\title{
A Study of the Association between Psychotropic Drugs and Cognitive Dysfunction in Patients with Schizophrenia
}

\author{
Aditi Chaudhari ${ }^{1}$, Mazumdar Kaustubh², Shobha Nair ${ }^{3}$, S. Anukarthika ${ }^{4}$, Divya Ramadas ${ }^{5}$ \\ ${ }^{1}$ Consultant Psychiatrist, \\ ${ }^{2}$ Former Consultant and Medical Officer In-charge and Former Head, Medical Division, BARC Hospital, \\ ${ }^{3}$ Consultant Psychiatrist and Head, \\ ${ }^{4}$ Third year DNB Resident, \\ ${ }^{5}$ Clinical Psychologist, \\ Department of Psychiatry, B.A.R.C. Hospital, Anushaktinagar, Mumbai. \\ Corresponding Author: Aditi Chaudhari \\ Email - aditidagaonkar@gmail.com / draditi@barc.gov.in
}

\begin{abstract}
Background: Cognitive deficits among patients with schizophrenia are now recognized as being widely prevalent and one of the most disabling aspects of the illness, as they are associated with poor functional outcomes. Psychotropic medications and benzodiazepines, which are often used in these patients could impact cognition as could the extrapyramidal side-effects. Yet, here are hardly any Indian studies on the subject. The aim of this study was to determine whether cognitive dysfunction among patients with schizophrenia is associated with extrapyramidal symptoms, anticholinergic burden of psychotropic drugs and benzodiazepine dosage

Methodology: 40 clinically stable, out-patients suffering from schizophrenia, without any pre-existing neurological disorders between the ages of 18 to 60 years, who had been compliant with medications were selected. Extrapyramidal symptoms (EPS) were assessed using the modified Simpson-Angus scale, anticholinergic burden (ACB) was evaluated using the Anticholinergic burden scale. The benzodiazepine dosage was noted. Cognition was assessed using the Stroop Color and Word Test and the Wisconsin Card sorting test. The correlations between scores on EPS and ACB and neuropsychological tests were carried out using partial correlations controlling for positive and negative symptoms.

Results: There were no significant associations noted between extrapyramidal symptoms, anticholinergic burden, benzodiazepine dosage and performance on the neurocognitive tests used.

Conclusion: Methodological differences make it difficult to construct comparisons across studies but there is some evidence to support our findings.
\end{abstract}

Keywords: Schizophrenia, extra-pyramidal symptoms, anticholinergic burden, benzodiazepines, cognition.

(Paper received $-8^{\text {th }}$ December 2019, Peer review completed $-24^{\text {th }}$ December 2019)

(Accepted $-27^{\text {th }}$ December 2019)

\section{INTRODUCTION}

It is well documented that cognitive deficits among patients with schizophrenia are widely prevalent and one of the most disabling aspects of the illness, as they are associated with unfavourable functional outcomes [1]. Inferences about a patient's cognitive impairments are characteristically made according to their performance on standardised cognitive tests [2]. Antipsychotic medications form the mainstay of pharmacological interventions in schizophrenia, whereas concomitant medications such as antidepressants, mood stabilizers, and anxiolytics are not uncommon [3]. It is possible that some of these drugs may have an adverse impact on cognition. Two mechanisms may explain cognitive dysfunction under antipsychotic 
medication [4]. One is the dopamine receptor blockade. PET studies have shown that a dysregulation in the dopamine synthesis in the striatum and and prefrontal D1 receptor and extra- striatal D2/D3 receptor availability may lead to cognitive dysfunction in schizophrenia [5]. There is also evidence that drug induced Parkinson's disease or extrapyramidal symptoms (EPS), which are the very common side effects of conventional antipsychotics are associated with a poor performance on neuropsychological tests [6-7]. Some authors propose that this link is mediated by the slow motor speed which further translates into poor performance on the objective testing [2]. However, certain studies have also shown that the relationship between EPS and neuropsychological deficits was not accounted for by slowed motor or mental processing and the pathophysiological mechanisms underlying the two phenomena may overlap in schizophrenia [6]. A second mechanism is the effect of cholinergic blockade. A review by Sarter and others, states that extensive research in basic sciences in the form of animal studies and psychopharmacological studies in humans and studies using functional imaging techniques have shown that the cortical cholinergic input systems are major modulators of the brain's attentional system [8]. Another review on the subject has reported strong evidence for the role of muscarinic and nicotinic acetylcholine receptors in cognitive impairment in schizophrenia [9] . Most antipsychotic medications possess anticholinergic properties [10]. The side-effect profile of antipsychotic medication is also sometimes treated with anticholinergic agents. This creates a considerable anticholinergic burden that impairs cognition and functional capacity [11-13]. Studies have investigated the relationship between benzodiazepine consumption and cognitive decline and found it to be significant following both acute and long-term usage [14].

Despite the increased interest in cognitive impairment in schizophrenia and its possible association with psychotropic medication, there are hardly any Indian studies on this subject. This gap of knowledge is of concern because pharmacological treatments when aggregated may further worsen cognition amongst these patients who are already impaired due to the primary disease process itself. This is the background of our study which was exploratory in nature with no a priori hypothesis.

\section{METHODOLOGY}

\section{Research Question}

What is the association between anti-psychotics, benzodiazepine use and extra pyramidal side-effects and cognitive dysfunction in patients with schizophrenia?

\section{Aims and Objectives}

To determine whether cognitive dysfunction among patients with schizophrenia is associated with

1. Extrapyramidal symptoms (EPS)

2. Anticholinergic burden (ACB)

3. Benzodiazepine dosage

\section{Sample selection and Methods}

The study was conducted at the out-patient unit of the Department of Psychiatry, at a Government Teaching Hospital. Approval from the Institutional Ethics Committee was obtained. (Reference No: BHMEC/ NP/ 01/18). The design of the study was cross-sectional. The study was conducted in January-February 2018. The calculation of the sample size was done using OpenEpi version 3.0. [15]. As seen in some past Indian studies [16], the proportion of patients of schizophrenia with cognitive impairment was estimated at $70 \%$. Using a confidence level of $95 \%$ and an absolute precision level of $10 \%$, we obtained a sample of 38 , out of the 70 patients we expected to seek within the age group of $18-60$ years. We rounded this up to 40 . The study sample was to reflect patients who were judged as clinically stable by the treating psychiatrist, who were living in their homes. So, patients between the ages of 18 and 60 years, who had been diagnosed with schizophrenia of any type as per International Classification of Diseases - 10 criteria with a duration of illness more than 2 years were included. They had been compliant with medications and this was assessed by the report of a reliable caregiver and also by scrutiny of the follow up schedule of the patient to collect his / her medications. The patients had not required any change in medications in the past three months. The patients who were excluded were those who required hospitalization for any reason at the time of assessment 
as well as those who suffered from any co-morbid psychiatric disorder except for nicotine dependence, Also, patients who had received electro-convulsive therapy in the past six months and those with co-morbid mental retardation, neurological disorders, blindness of any type and deafness were excluded. Other studies have used similar recruitment criteria [17-18]. The patients were assessed for current symptom severity, extrapyramidal symptoms, anticholinergic burden and benzodiazepine dosage. Neuropsychological assessment was carried out using the Stroop Colour and Word Test and Wisconsin Card Sorting Test. Convenience sampling was used.

\section{Instruments}

1. A semi-structured proforma was designed to obtain the various socio-demographic and illness related variables.

2. Positive and Negative Syndrome Scale for Schizophrenia [19] was used to rate the current severity of schizophrenia.

3. Modified Simpson-Angus scale (MSAS) [20]: Extrapyramidal symptoms (EPS), as caused by neuroleptics, were rated using the modified Simpson- Angus scale. It indexes ten Parkinsonian symptoms on a five-point scale (0-4). Scores are derived by adding item scores and the severity of EPS is graded as normal, minimal, clinically significant and severe depending upon the cut-offs provided.

4. Anticholinergic Cognitive Burden Scale [21]: Anticholinergic burden (ACB) was assessed using the Anticholinergic Cognitive Burden (ACB) scale. The ACB scale was developed by a multidisciplinary expert panel based on a systematic review of medications with known anticholinergic activity likely to have an effect on cognition. It contains 88 listed medications. Each listed medication can be rated on a 4-point scale (0-3): $0=$ no anticholinergic activity, $1=$ mild anticholinergic activity, $2=$ moderate anticholinergic activity, and $3=$ severe anticholinergic activity. Drugs with possible anticholinergic effects are defined as those with serum anticholinergic activity or in vitro affinity for muscarinic receptors but no known clinically relevant cognitive effects (ACB score 1). Drugs with established, clinically relevant cognitive effects are considered definitely anticholinergic (ACB score 2 or 3) [22]. The total anticholinergic burden is then calculated by aggregating the score for each listed medication. The ACB scale has been previously validated in this population [23].

5. The number of patients who were on benzodiazepines was noted. The dose was converted into an equivalent dose of diazepam and recorded [24].

\section{Neuropsychological assessment}

1. The Stroop Colour and Word Test (Stroop Test) [25]: This is an extensively used neuropsychological test which assesses the ability to inhibit cognitive interference. Previous literature also reports its application to measure other cognitive functions such as attention, processing speed, cognitive flexibility and working memory [24]. It was administered manually, in English and scoring was done depending upon the errors committed.

2. Wisconsin Card Sorting Test (WCST) [26]: This was used a measure of abstract reasoning, executive functioning and problem solving.

\section{STATISTICAL ANALYSIS}

The data analysis has been carried out using the IBM SPSS 20 Software. The socio-demographic and illness related variables have been expressed in terms of frequency and percentages. The scores on the scales have been mentioned as mean scores with standard deviation. The scores on the scales have been correlated with the scores on the neuropsychological tests, controlling for the scores on positive and negative symptoms, using the partial correlation of the Pearson's Correlation Coefficient Test. The data was assessed for normative distribution and both parametric and non- parametric tests for comparison. Lastly, to consider the role of other confounding factors, they were entered in a step-wise linear regression model as predictors for cognitive dysfunction. A $\mathrm{p}$ value of less than 0.05 was considered to be statistically significant. 


\section{RESULTS}

\section{Socio-demographic and Clinical Profile}

A total of 40 patients with schizophrenia were studied of which 15 were males and 25 were females. The socio-demographic and clinical profile of the patients was as shown in table 1.

Table 1: Socio-demographic and Clinical Profile

\begin{tabular}{|lll|}
\hline Domain & Frequency & Percentage \\
\hline Age & & \\
$18-45$ years & 26 & 65 \\
46-60 years & 14 & 35 \\
\hline Education & & \\
Upto Class 10 & 10 & 25 \\
Upto Class 12 & 12 & 30 \\
Graduate & 9 & 22.5 \\
Post Graduate & 9 & 22.5 \\
\hline Occupation & & \\
Employed & 11 & 27.5 \\
Unemployed & 13 & 32.5 \\
Housewives & 16 & 40 \\
\hline Duration of illness & & \\
2-5 years & 11 & 27.5 \\
5-10 years & 12 & 30 \\
$>10$ years & 17 & 42.5 \\
\hline Nicotine Use & & \\
Males & 6 & 40 \\
Females & 2 & 8 \\
\hline
\end{tabular}

\section{Symptom Profile}

The scores on the positive syndrome subscale ranged from 7 to 20 with a mean score of $10.90 \pm 3.82$ and median score was 9 while those on the negative syndrome subscale ranged from 7 to 32 with a mean score of $14.10 \pm 6.12$ and a median score of 12 . Thus, the scores on the negative syndrome subscale were significantly higher than those on the positive syndrome subscale. (Wilcoxon Signed Ranks Test: $Z=-4.055$; $\mathrm{p}=0.0001)$.

\section{Pharmacological Profile}

$4(10 \%)$ patients were only on typical antipsychotics, $18(45 \%)$ were only on atypical antipsychotics and 18 (45\%) were on combination therapy. $14(35 \%)$ patients were receiving depot preparations, all of which were first generation. 4 patients i.e. $10 \%$ were found to be on prescription benzodiazepines.

\section{Extrapyramidal symptom scores}

The scores on the Modified Simpson Angus Scale (MSAS) for extrapyramidal symptoms (EPS) ranged from 0 to 7 . The median score was 2 and the mean scores were $2.40 \pm 2.04$. This corresponds to a normal score. The severity of EPS was as shown in figure 1 .

The patients only on typical antipsychotics had the highest scores on EPS $(3.50 \pm 2.51)$ followed by those on combination therapy $(3.16 \pm 2.06)$. The EPS scores were the lowest for patients only on atypical antipsychotics $(1.38 \pm 1.50)$. The groups differed significantly. (Kruskal-Wallis test: Chi-square $=8.235 ; \mathrm{df}$ $=2, p=0.016)$. Post hoc serial Mann-Whitney U test showed that patients on combination regimen had significantly higher EPS as compared to patients only on atypical antipsychotics. (Mann-Whitney $U=77.00$; Wilcoxon $\mathrm{W}=248.00, \mathrm{Z}=-2.751, \mathrm{p}=0.006$ ). 
Figure 1: Scores on the Modified Simpson Angus Scale

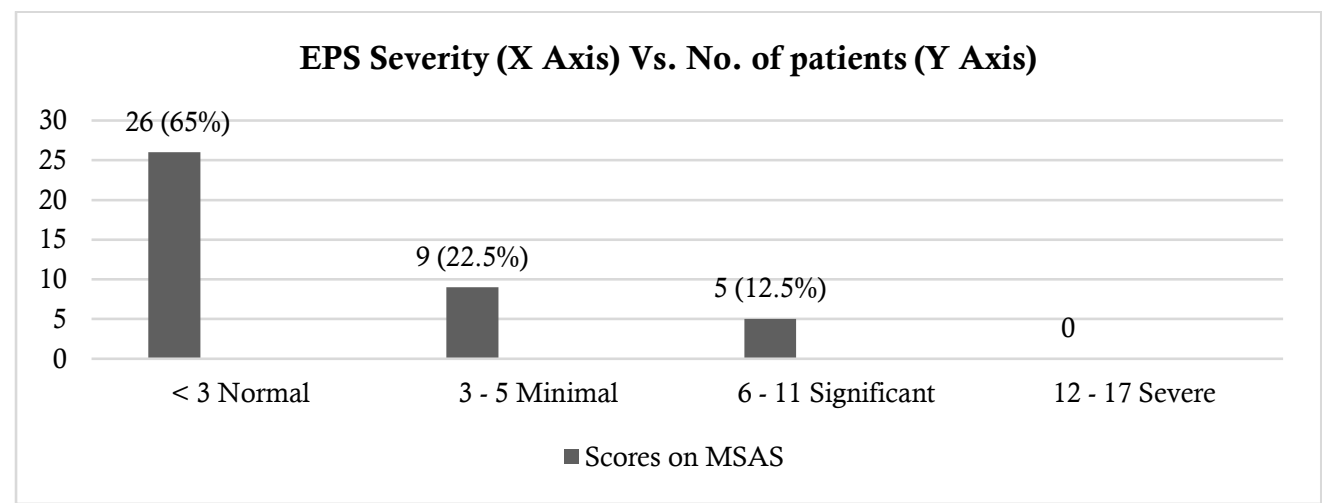

\section{Anticholinergic Burden Scores}

The mean scores on the Anticholinergic Burden Scale (ACB) were $5.86 \pm 2.89$.

The scores of the patients were as shown in the figure 2 .

Figure 2: Scores on the Anticholinergic Burden Scale

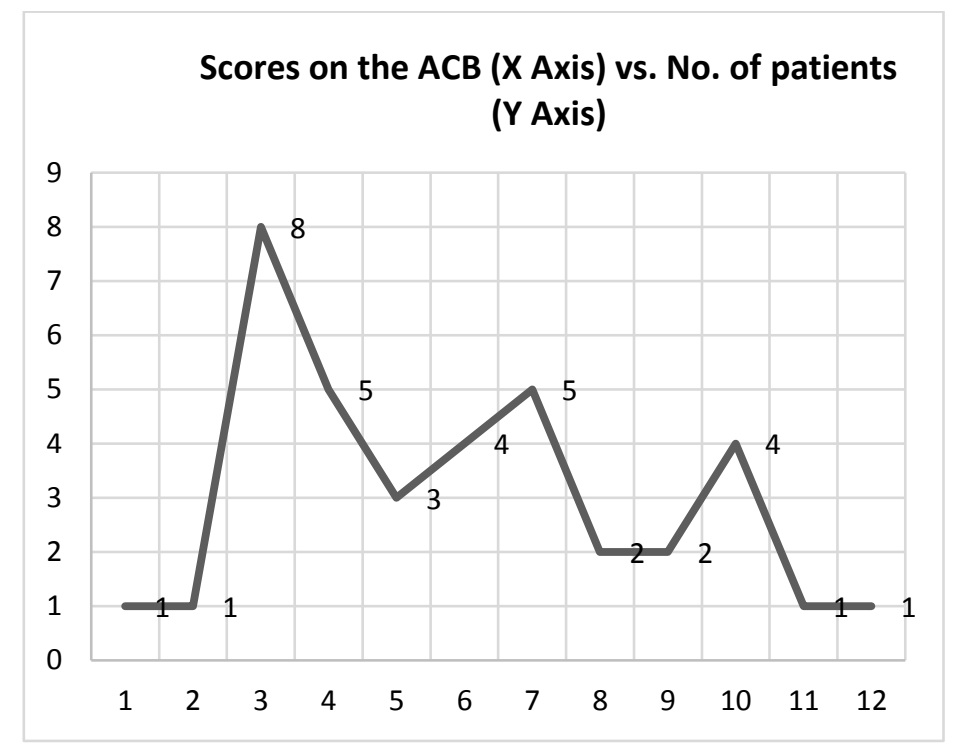

Patients who were on combination of typical and atypical antipsychotics had a higher anticholinergic burden score $(7.83 \pm 2.59)$ as compared to those only on typicals $(3.75 \pm 3.86)$ or atypicals $(4.33 \pm 1.68)$ This difference was statistically significant. [ $F=11.59$; $\mathrm{df}(2,37) ; \mathrm{p}=0.001]$.

Although patients receiving depot injections had higher ACB scores $(6.78 \pm 3.53)$ than those not taking them, $(5.34 \pm 2.49)$, this difference was not statistically significant. $[t=1.50 ; \mathrm{df}=38 ; \mathrm{p}=0.142]$.

\section{Equivalent Benzodiazepine Doses}

The mean diazepam equivalent dose for the four patients who were taking benzodiazepines was $15 \mathrm{mg}$.

\section{Neuropsychological Profiles}

\section{Stroop Test}

The scores on the Stroop test ranged from 27 to 110 with a mean score of $63.82 \pm$ S.D. $=21.07$. Overall 33 (82.5\%) patients had results suggestive of impairment. [Norms: For age group 18 to 49 years a raw score of 98 and below whereas for the age group $>50$ years, a score below 61 indicates dysfunction. Decreasing score 
is thus inversely proportional to dysfunction]. 22 out of the $26(84.6 \%)$ patients in the age group of 18 to 45 years while 11 out of the $14(78.57 \%)$ patients in the age group of 46 to 60 years indicated dysfunction on this test. This difference was not statistically significant. (Pearson Chi Square $=0.230 ; \mathrm{df}=1$; Fisher's Exact Test Sig. 0.679).

\section{Wisconsin Card Sorting Test (WCST)}

The T scores on the Wisconsin card Sorting Test ranged from 19 to 53 with a median score of 31.00 and a mean score of $31.67 \pm$ S.D. $=9.11$, which is indicative of borderline performance.

The performance of $20 \%$ of the patients was within the normal range whereas $37.5 \%, 17.5 \%$ and $25 \%$ had borderline, deficient and significantly deficient performance respectively.

\section{Pharmacological correlates of Cognition}

\section{Extrapyramidal symptoms, Anticholinergic Burden and Cognition}

The scores on the two neuropsychological tests were correlated with the scores for extrapyramidal symptoms and anticholinergic burden, while controlling for the scores on the positive and negative symptoms, using partial correlations. No significant correlations were noted as seen in table 2 .

Table 2: Partial correlations between scores on neuropsychological testing and scores on extrapyramidal symptoms and anticholinergic burden

\begin{tabular}{|lll|}
$\begin{array}{l}\text { Control variables } \\
\text { Positive and Negative }\end{array}$ & $\begin{array}{l}\text { Extrapyramidal } \\
\text { symptoms (Score on } \\
\text { MSAS) }\end{array}$ & $\begin{array}{l}\text { Anticholinergic burden } \\
\text { (Score on ACB) }\end{array}$ \\
\hline Stroop test & & -0.023 \\
Correlation & -0.220 & 0.889 \\
Significance (2 tailed) & 0.185 & 36 \\
Df & 36 & \\
\hline Wisconsin Card Sorting test & & -0.061 \\
Correlation & -0.003 & 0.715 \\
Significance (2 tailed) & 0.987 & 36 \\
df & 36 & \\
\hline
\end{tabular}

\section{Benzodiazepine dosage and Cognition}

The mean scores on the Stroop test $(63.75 \pm 9.53)$ and those on the WCST $(28.00 \pm 4.69)$ for the four patients who were on prescription benzodiazepines were lower than for the patients who were not on this group of drugs. [(Stroop Test: $63.83 \pm 22.06)$; (WCST: $32.08 \pm 9.43)$ ]. However, this difference was not statistically significant as shown in table 3 and 4.

Table 3: Comparison of scores on Stroop Test between patients not taking and taking benzodiazepines

\begin{tabular}{|c|c|c|c|c|c|}
\hline & \multirow[t]{2}{*}{$\mathbf{t}$} & \multirow[t]{2}{*}{ df } & \multirow{2}{*}{$\begin{array}{l}\text { Sig ( } 2 \\
\text { tailed) }\end{array}$} & \multicolumn{2}{|c|}{$95 \%$ Confidence interval of the Difference } \\
\hline & & & & Lower & Upper \\
\hline Stroop Test & 0.007 & 38 & 0.994 & -22.69 & 2.86 \\
\hline
\end{tabular}


Table 4: comparison of scores on WCST between patients not taking and taking benzodiazepines

\begin{tabular}{|ll|}
\hline & W Score \\
\hline Mann - Whitney U & 54.500 \\
Wilcoxon W & 64.500 \\
Z & -0.791 \\
p value & 0.429 \\
Exact Sig. [ 2* (1 - tailed Sig)] & 0.445 \\
\hline
\end{tabular}

\section{Grouping variable: Benzodiazepines}

To study the effects of other variables viz. age, gender, education, occupation, nicotine use and duration of illness on neuropsychological test performance, they were entered as predictors along with the positive and negative symptom score, extrapyramidal symptom score, anticholinergic burden and benzodiazepine use, in a stepwise linear regression model. For the scores on the Stroop Test, the prediction model contained only one predictor and was reached in one step with no variable removed. The model was statistically significant, $(\mathrm{F}(1,38)=7.908, \mathrm{p}=0.008)$ and accounted for approximately $17 \%$ variance of scores. $(\mathrm{R} 2=0.172$, Adjusted $\mathrm{R} 2=0.150)$. It was found that only increased age significantly predicted poor performance $(\beta=-0.415 ; \mathrm{t}=$ -2.812; $\mathrm{p}=0.008)$. For the normalised scores on the WCST, the prediction model contained only one predictor and was reached in one step with no variable removed. The model was statistically significant ( $F$ $(1,38)=4.755, \mathrm{p}=0.035)$ but accounted for approximately $11 \%$ variance of scores. $(\mathrm{R} 2=0.111$, Adjusted $\mathrm{R} 2=0.088)$. It was found that only negative symptom scores significantly predicted poor performance $(\beta=$ $-0.333 ; \mathrm{t}=-2.181 ; \mathrm{p}=0.035)$

\section{DISCUSSION}

The purpose of the present study was to determine the association between performance on cognitive tests and the pharmacological profile in stable patients with schizophrenia living in the community. However, there are a number of limitations to the generalizability of our findings. Any study on cognition in schizophrenia is challenged by multiple confounding factors such as pre-morbid intelligence and functioning, co-morbid depressive and obsessive symptoms and rehabilitative services. Inclusion of all these factors was beyond the scope of the study. The stringent inclusion and exclusion criteria have resulted in a smaller sample size. Numbers of patients taking benzodiazepines were very small. Our study included only clinically stable, compliant, patients, living at home. Therefore, any change in the severity of their illness or compliance could impact their cognitive performance. This study also has cross sectional design. Hence the findings of the present study need to be interpreted in the context of these limitations.

$82.5 \%$ of the patient scored poorly on the Stroop test indicating deficiencies in attention, processing speed, cognitive flexibility and working memory apart from cognitive interference. An equally high number i.e. $80 \%$ indicated below normal performance on the WCST reflecting the high prevalence of compromised executive function. Our findings concur with an extensive amount of literature $[1,9,13,27]$ which establishes cognitive impairment in schizophrenia to be a highly prevalent phenomenon. The present study showed no significant association between extrapyramidal symptoms, anticholinergic burden, benzodiazepine dosage and the neurocognitive domains studied.

Our study differs from some studies [2,6-7] that have reported a significant association between EPS and cognitive dysfunction. However, a careful scrutiny of the sample selection in all these studies can account for these differences. The patients included in the study by Fervaha and others [2] were not on antipsychotics at the time of assessment. The authors themselves posit that the presence of EPS or cognitive impairment cannot be reliably ascribed to psychotropic agents. The study by Krausz and others [7] studied in-patients within a week or two of hospitalisation while Palmer and others [6] included only patients over the age of 45. Thus, the confounding effects of acute illness and age cannot be ruled out. Only two studies [17-18] had a respondent group similar to ours. Out of these the study by Tanaka and others [17] reported that, although 
the severity of extrapyramidal symptoms in their study was quite low, it still had a significant association with cognitive dysfunction. In our study as well, only 5 (12.5\%) patients had significant EPS and this group did have the least score on both the cognitive tests albeit this did not attain statistical significance. Yje second study by Potvin and others [18] reported that anti-psychotic induced parkinsonism showed a significant association with working memory deficits but not with the other cognitive domains studied. However, these deficits were also associated with the patient's age. This illustrates that the interpretation of the findings of studies that vary in design, patient-selection criteria, instruments and scoring methods requires considerable caution. Multiple confounding factors make drawing inferences, a challenging task. Investigations based on uniform methodologies could throw some light on this association.

Research on effect of anticholinergic burden on cognition has yielded conflicting findings. One of the most recent studies [3] on the subject has reported that although the anticholinergic burden was associated with poorer cognitive performance, this impact is likely to be of doubtful or minimal clinical significance. Another study also reported that anticholinergic load impaired declarative memory and divided attention but not intelligence, working memory, executive function, verbal and visual conceptual fluency or motor speed [28]. The authors concluded that anticholinergic load is not associated with general cognitive impairment. However, some studies have demonstrated that anticholinergic activity is associated with impaired performance on neuropsychological tests [13] and interfere with ability to benefit from cognitive and psychosocial treatment programmes [11].

Research [28] shows that the ascending cholinergic cortical projections from the nucleus basalis of Meynert plays a key role in the cognitive processes of the human brain. The two receptor subtypes found in these projections are nicotinic and muscarinic. The distribution and cellular effects associated with the activation of these receptors differ considerably. Some authors [28] have postulated that while psychotropic agents do have anti muscarinic activity, they cannot be said to have any major actions at the nicotinic receptors. Whether anti-muscarinic and anti-nicotinic actions have any differential effects on cognition was beyond the scope of the present study but this concept does provide a direction for future research.

Another observation in the present study was that only four patients i.e. $10.8 \%$ were on prescription benzodiazepines. It is obvious that this number is too small to make any reasonable inferences. Although not statistically significant, these patients did have lower scores on the neuropsychological tests. A metaanalysis [29] on this subject, postulates that benzodiazepine users do have cognitive impairment as compared to controls but this may be partially reversible on stopping the benzodiazepines.

Finally, the stepwise linear regression analysis helped to adjust for the role of certain confounding variables such as age, gender, education, occupation, duration of illness and symptom severity. Increased age was associated with a poorer performance on the Stroop Test. Patients over the age of 45 years had significantly lower scores on the Stroop $(52.64 \pm 17.14)$ test as compared to younger patients $(69.84 \pm 20.78)(t=2.645$; $\mathrm{df}=38 ; \mathrm{Sig}(2$ tailed $)=0.012)$. A systematic review [30] on Stroop interference, practice, and aging provides robust evidence in support of this finding. The authors have hypothesised the role of frontal lobe dysfunction and inhibition deficit to explain this difference [30].

Negative symptom score was associated with a poor score on the WCST. A recent Indian study has also reported that patients with negative symptoms of schizophrenia scored significantly lower on the WCST as compared to those with positive symptoms and controls [31]. There are also some body of evidence which suggests that negative symptoms appear to play an indirect role, mediating the relationship between neurocognition and social cognition with functional outcomes [32]. Such patients thus appear to be doubly disadvantaged and perhaps maximally in need for targeted interventions. However, although our findings are in tune with the existing literature, it was observed that only $11 \%$ variance could be explained by this result. In a conceptual overview on this subject, Harvey and others [33] have addressed the possible relationship between negative and cognitive symptoms using four different models and have concluded that the two symptoms may be separable if not conceptually independent domains of the illness.

Nicotinic cholinergic receptors have been implicated in cognitive dysfunction in schizophrenia [34] and the higher prevalence of smoking among schizophrenia patients has been attributed to the self-medication of these cognitive deficits. While certain studies report that schizophrenia patients who smoked, performed better on neuropsychological tests [34] but certain other authors have reported that nicotine affected only attention but there was no effect on learning, memory, language or visuospatial and construction abilities 
[35]. This is partially in keeping with our findings. The effects of nicotine thus do not extend to all areas of cognition [35] but even this differential effect has been hypothesised to influence the pattern and persistence of smoking in schizophrenia [35].

\section{CONCLUSIONS}

- $82.5 \%$ of the patients had deficiencies in attention, processing speed, cognitive flexibility, working memory and cognitive interference as seen from their performance on the Stroop Test.

- $80 \%$ of the patients exhibited executive dysfunction as evidenced from the Wisconsin card sorting test.

- There was no significant association between extrapyramidal symptom severity, anticholinergic burden and benzodiazepine dosage and the cognitive domains studied.

\section{REFERENCES}

1. Green MF, Kern RS, Heaton RK. Longitudinal studies of cognition and functional outcome in schizophrenia: implications for MATRICS. Schizophr Res 2004;72(1):41-51.

2. Fervaha G, Agid O, Takeuchi H, Lee J, Foussias G, Zakzanis KK, Graff-Guerrero A, Remington G. Extrapyramidal symptoms and cognitive test performance in patients with schizophrenia. Schizophr Res 2015;161(2-3):351-6.

3. San Ang M, Rashid NA, Lam M, Rapisarda A, Kraus M, Keefe RS, Lee J. The impact of medication anticholinergic burden on cognitive performance in people with schizophrenia. J Clin Psychopharmacol 2017;37(6):651-7.

4. Rehse M, Bartolovic M, Baum K, Richter D, Weisbrod M, Roesch-Ely D. Influence of antipsychotic and anticholinergic loads on cognitive functions in patients with schizophrenia. Schizophr Res Treat 2016;Article ID 8213165.

5. Takano H. Cognitive function and monoamine neurotransmission in schizophrenia: evidence from positron emission tomography studies. Front Psychiatry 2018;9:228.

6. Palmer BW, Heaton RK, Jeste DV. Extrapyramidal symptoms and neuropsychological deficits in schizophrenia. Biol Psychiatry 1999;45(6):791-4.

7. Krausz M, Moritz SH, Naber D, Lambert M, Andresen B. Neuroleptic-induced extrapyramidal symptoms are accompanied by cognitive dysfunction in schizophrenia. Eur Psychiatry 1999;14(2):84-8.

8. Sarter M, Lustig C, Taylor SF. Cholinergic contributions to the cognitive symptoms of schizophrenia and the viability of cholinergic treatments. Neuropharmacol 2012;62(3):1544-53.

9. Goff DC, Hill M, Barch D. The treatment of cognitive impairment in schizophrenia. Pharmacol Biochem Behav 2011;99(2):245-53.

10. Chew ML, Mulsant BH, Pollock BG, Lehman ME, Greenspan A, Mahmoud RA, Kirshner MA, Sorisio DA, Bies RR, Gharabawi G. Anticholinergic activity of 107 medications commonly used by older adults. J Am Geriatr Soc 2008;56(7):1333-41.

11. O'Reilly K, O'Connell P, Donohoe G, Coyle C, O'Sullivan D, Azvee Z, Maddock C, Sharma K, Sadi H, McMahon M, Kennedy HG. Anticholinergic burden in schizophrenia and ability to benefit from psychosocial treatment programmes: a 3-year prospective cohort study. Psychol Med 2016;46(15):3199-211.

12. Spohn HE, Strauss ME. Relation of neuroleptic and anticholinergic medication to cognitive functions in schizophrenia. J Abnorm Psychol 1989;98(4):367.

13. Vinogradov S, Fisher M, Warm H, Holland C, Kirshner MA, Pollock BG. The cognitive cost of anticholinergic burden: decreased response to cognitive training in schizophrenia. Am J Psychiatry 2009;166(9):1055-62.

14. Boeuf-Cazou O, Bongue B, Ansiau D, Marquié JC, Lapeyre-Mestre M. Impact of long-term benzodiazepine use on cognitive functioning in young adults: the VISAT cohort. Eur J Clin Pharmacol 2011;67(10):1045-50.

15. Dean AG, Sullivan KM, Soe MM. OpenEpi: open source epidemiologic statistics for public health, version.

16. Talreja BT, Shah S, Kataria L. Cognitive function in schizophrenia and its association with sociodemographics factors. Industr Psychiatr J 2013;22(1):47-55.

17. Tanaka T, Tomotake M, Ueoka Y, Kaneda Y, Taniguchi K, Nakataki M, Numata S, Tayoshi S, Yamauchi $\mathrm{K}$, Sumitani S, Ohmori T. Clinical correlates associated with cognitive dysfunction in people with schizophrenia. Psychiatr Clin Neurosci 2012;66(6):491-8. 
18. Potvin S, Aubin G, Stip E. Antipsychotic-induced parkinsonism is associated with working memory deficits in schizophrenia-spectrum disorders. Eur Arch Psychiatr Clin Neurosci 2015;265(2):147-54.

19. Kay SR, Fiszbein A, Opler LA. The positive and negative syndrome scale (PANSS) for schizophrenia. Schizophr Bull 1987;13(2):261-76.

20. Simpson GM, Angus JW. A rating scale for extrapyramidal side effects. Acta Psychiatr Scand 1970;45(S212):11-9.

21. Campbell N, Boustani M, Limbil T, Ott C, Fox C, Maidment I, Schubert CC, Munger S, Fick D, Miller D, Gulati R. The cognitive impact of anticholinergics: a clinical review. Clin Interv Aging 2009;4:225-33.

22. Pasina L, Djade CD, Lucca U, Nobili A, Tettamanti M, Franchi C, Salerno F, Corrao S, Marengoni A, Iorio A, Marcucci M. Association of anticholinergic burden with cognitive and functional status in a cohort of hospitalized elderly: comparison of the anticholinergic cognitive burden scale and anticholinergic risk scale. Drugs Aging 2013;30(2):103-12.

23. Salahudeen MS, Duffull SB, Nishtala PS. Anticholinergic burden quantified by anticholinergic risk scales and adverse outcomes in older people: a systematic review. BMC Geriatrics 2015;15(1):31.

24. Taylor DM, Barnes TR, Young AH. The Maudsley prescribing guidelines in psychiatry. John Wiley \& Sons; 2018.

25. Kane MJ, Engle RW. Working-memory capacity and the control of attention: the contributions of goal neglect, response competition, and task set to Stroop interference. J Experiment Psychol Gen 2003;132(1):47-54.

26. Heaton RK. Wisconsin card sorting test manual. Psychological Assessment Resources. 1981.

27. Weiser M, Shneider-Beeri M, Nakash N, Brill N, Bawnik O, Reiss S, Hocherman S, Davidson M. Improvement in cognition associated with novel antipsychotic drugs: a direct drug effect or reduction of EPS ?. Schizophr Res 2000;46(2-3):81-9.

28. Minzenberg MJ, Poole JH, Benton C, Vinogradov S. Association of anticholinergic load with impairment of complex attention and memory in schizophrenia. Am J Psychiatry 2004;161(1):116-24.

29. Barker MJ, Greenwood KM, Jackson M, Crowe SF. Persistence of cognitive effects after withdrawal from long-term benzodiazepine use: a meta-analysis. Arch Clin Neuropsychol 2004;19(3):437-54.

30. Davidson DJ, Zacks RT, Williams CC. Stroop interference, practice, and aging. Aging Neuropsychol Cogn 2003;10(2):85-98.

31. Singh S, Aich TK, Bhattarai R. Wisconsin Card Sorting Test performance impairment in schizophrenia: An Indian study report. Indian J Psychiatry 2017;59(1):88-92.

32. Madeira N, Caldeira S, Bajouco M, Pereira AT, Martins MJ, Macedo A. Social Cognition, Negative Symptoms and Psychosocial Functioning in Schizophrenia. International Journal of Clinical Neurosciences and Mental Health 2016;3:1.

33. Harvey PD, Koren D, Reichenberg A, Bowie CR. Negative symptoms and cognitive deficits: what is the nature of their relationship?. Schizophr Bull 2005;32(2):250-8.

34. Beck AK, Baker AL, Todd J. Smoking in schizophrenia: cognitive impact of nicotine and relationship to smoking motivators. Schizophr Res: Cogn 2015;2(1):26-32.

35. Harris JG, Kongs S, Allensworth D, Martin L, Tregellas J, Sullivan B, Zerbe G, Freedman R. Effects of nicotine on cognitive deficits in schizophrenia. Neuropsychopharmacology 2004;29(7):1378-86.

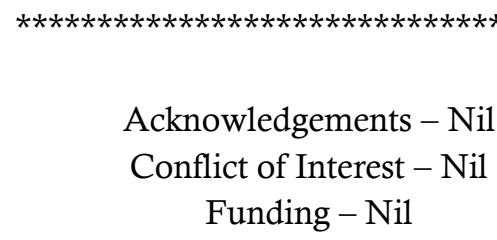

Indian Journal of Mental Health 2019;6(4) 